\title{
Photon Bunching in a Rotating Reference Frame
}

\author{
Sara Restuccia, ${ }^{1, *}$ Marko Toroš, ${ }^{2,3, *}$ Graham M. Gibson, ${ }^{1}$ Hendrik Ulbricht, ${ }^{2}$ Daniele Faccio, ${ }^{1, \dagger}$ and Miles J. Padgett ${ }^{1, \sharp}$ \\ ${ }^{1}$ School of Physics and Astronomy, University of Glasgow, Glasgow G12 8QQ, United Kingdom \\ ${ }^{2}$ Department of Physics and Astronomy, University of Southampton, Southampton SO17 1BJ, United Kingdom \\ ${ }^{3}$ Department of Physics and Astronomy, University College London, London WC1E 6BT, United Kingdom
}

(Received 18 May 2019; published 10 September 2019)

\begin{abstract}
Although quantum physics is well understood in inertial reference frames (flat spacetime), a current challenge is the search for experimental evidence of nontrivial or unexpected behavior of quantum systems in noninertial frames. Here, we present a novel test of quantum mechanics in a noninertial reference frame: we consider Hong-Ou-Mandel (HOM) interference on a rotating platform and study the effect of uniform rotation on the distinguishability of the photons. Both theory and experiments show that the rotational motion induces a relative delay in the photon arrival times at the exit beam splitter and that this delay is observed as a shift in the position of the HOM dip. This experiment can be extended to a full general relativistic test of quantum physics using satellites in Earth's orbit and indicates a new route toward the use of photonic technologies for investigating quantum mechanics at the interface with relativity.
\end{abstract}

DOI: 10.1103/PhysRevLett.123.110401

Introduction.-Quantum mechanics and general relativity, two cornerstones of modern physics, are separately well formalized and tested to great precision. Yet the reconciliation of the two in a unified theory has been, and remains, one of the open problems of theoretical physics. The difficulty in tackling this problem is twofold. First, the mathematical frameworks of these two theories, as well as their conceptual underpinnings, seem to be incompatible with each other [1]. Second, exploring regimes where both quantum mechanical and relativistic effects are important has proven to be a significant experimental challenge [2].

These difficulties have led to the pursuit of several approaches [3]. Particularly noteworthy is the framework of quantum field theory in curved spacetime [4,5], in which some far-reaching progress has been made, with the best known example being Hawking's prediction that black holes emit black-body radiation [6,7].

In view of the experimental challenges, several phenomenological models [2] have also been proposed, such as minimal length scale [8] and semiclassical models [9-14]. Although these models have the advantage of being testable, they still pose a significant experimental challenge [15].

Quantum mechanical experiments that probe gravity can be divided into (i) local and (ii) nonlocal classes. The former class probes special relativistic, noninertial effects in Minkowski spacetime, where the Riemann tensor corrections can be neglected, whereas the latter class, which has not yet been experimentally realized, would probe genuinely general relativistic effects, with the outcome of the experiment being related to the curvature of spacetime. So far there have been two experiments of type (i) neutron and atom interferometry [16-18], and entanglement witness of photon pairs [19]. Both of these experimental classes can be seen, using the equivalence principle, as being implemented either in a uniform gravitational field or in Rindler spacetime. There have been only a handful of proposals of type (ii), all of which are based on the same idea, namely that of one or more small masses interacting gravitationally [20-23]. The difficulty of type (ii) experiments can be appreciated by looking at the current experimental limits on the detection of weak gravitational forces $[24,25]$. These limits have led the community to also consider an indirect test, where the gravitational interaction, cumulatively over an interval of time, generates an entangled state [26,27].

In this Letter, we report a novel experiment of type (i) aimed at probing the behavior of entangled photons in a noninertial reference frame. Specifically, we demonstrate Hong-Ou-Mandel (HOM) interference [28] within a rotating reference frame.

Key to our experiments is the recognition that a HOM interference experiment is similar in configuration to a Sagnac interferometer. The latter is based on a ring cavity in which light coupled to the clockwise and anticlockwise paths interferes at the output. It is well known that rotation of the frame results in a phase shift in the interference and this effect is the basis of an optical gyroscope $[29,30]$. The HOM setup may also take the form of a ring cavity, but this time one in which the down-converted photons are created within the cavity and take clockwise and anticlockwise paths to interfere at the output. As mentioned above, rotation induces a phase shift between the clockwise and anticlockwise beams that is observed at the output of a generic interferometer (see Fig. 1). The question is whether the HOM dip undergoes a corresponding change. By measuring such a shift within a rotating frame, we obtain 
a relationship between the classical phase shift and the HOM path-length shift. In this way, we combine, in a single experiment, the relativistic Sagnac effect with a quantum mechanical HOM effect. This experiment also has the potential for generalization to a full general relativistic test of quantum physics using satellites in Earth's orbit, based on the gravitomagnetic clock effect [31].

Theory.-The rotating HOM interferometer is shown schematically in Fig. 1. A photon source generates a pair of indistinguishable photons that are separated from each other using a knife-edge prism and sent to the clockwise and anticlockwise paths within the interferometer. These photons are then recombined at a beam splitter. Whenever two indistinguishable photons combine at the beam splitter at the same time, quantum interference dictates that they will bunch and therefore always exit from the same output port (although which port this will be is completely random and cannot be known a priori). Because both photons are emitted from the same port, the detectors see a large decrease in coincidence counts compared with when the photons arrive at different times (when they do not bunch).

The experimental setup in Fig. 1 can be modeled using the theoretical description of the HOM interferometer [32], where the rotational motion of the platform generates a time delay in the arrival times of the two photons. This time delay introduces distinguishability between the two photons, which in turn modifies the interference at the output. We can estimate the time delay $\Delta t$ using the formula for Sagnac delay (which is a classical relativistic effect) at small rotational frequencies [29,30,33],
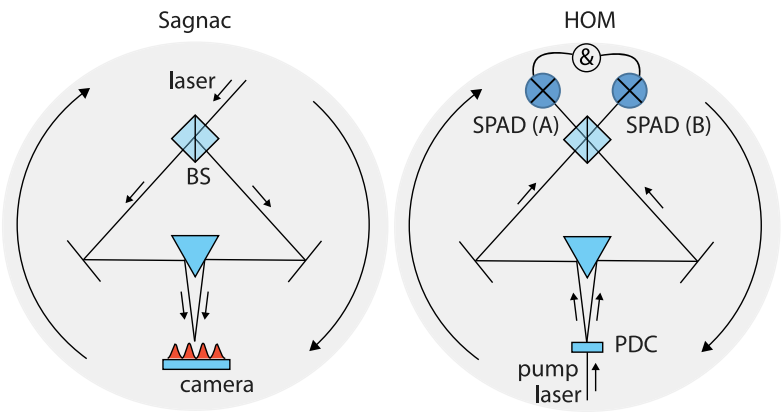

FIG. 1. Rotating interferometer schematic layouts: "Sagnac" indicates the classical interferometer formed from two counterpropagating beams that then overlap at a small angle on a camera that measures the resulting interference fringes. Platform rotation is detected as a lateral shift in the fringes. "HOM" indicates the quantum Hong-Ou-Mandel interferometer that can be implemented on the same platform as the classical Sagnac interferometer by introducing a down-conversion crystal in the plane of the camera. The two down-converted photons enter the interferometer and propagate in opposite directions, interfere at the beam splitter (BS), and are coincidence counted at the two BS output ports with single-photon avalanche detectors, SPADs (A) and (B). Rotation is detected by measuring a variation in the coincidence counts.

$$
\Delta t=8 \pi \frac{A f}{c^{2}},
$$

where $A$ is the area enclosed by the trajectories of the two photons and $f$ is the rotational frequency of the frame. The expression in Eq. (1) is the same for both the inertial, laboratory observer and the noninertial, corotating observer attached to the detector [34]: this is a consequence of the nonrelativistic motion of matter.

We consider a generic initial two-photon state

$\left|\chi_{i}\right\rangle=\int_{0}^{\infty} d \omega_{1} \int_{0}^{\infty} d \omega_{2} \psi\left(\omega_{1}, \omega_{2}\right) \hat{a}^{\dagger}\left(\omega_{1}\right) \hat{b}^{\dagger}\left(\omega_{2}\right)|0\rangle$,

where $\hat{a}\left(\omega_{1}\right)$ and $\hat{b}\left(\omega_{2}\right)$ denote the annihilation operators for modes of frequency $\omega_{1}$ propagating in the clockwise path and for modes of frequency $\omega_{2}$ propagating in the anticlockwise path, respectively, and $\psi$ is a $\mathbb{C}$-valued function. We now assume that the two-photon state, before the interaction with the beam splitter, evolves to

$\left|\chi_{e}\right\rangle=\int_{0}^{\infty} d \omega_{1} \int_{0}^{\infty} d \omega_{2} \psi\left(\omega_{1}, \omega_{2}\right) e^{-i \phi} \hat{a}^{\dagger}\left(\omega_{1}\right) \hat{b}^{\dagger}\left(\omega_{2}\right)|0\rangle$,

where $\phi$ is the accumulated phase, which depends on the experimental setup (e.g., on the length of the optical fiber). Specifically, we assume that the phase is given by $\phi\left(\omega_{1}, \omega_{2}, f\right)=\omega_{1}\left[t^{(+)}(f)+\delta t_{p}\right]+\omega_{2} t^{(-)}(f)$, where $t^{(+)}$ and $t^{(-)}$denote the times of flight from the source to the beam splitter for two classical light signals propagating in the clockwise and anticlockwise directions, respectively. $\delta t_{p}$ denotes an additional time delay due to differences in the optical path between the two interferometer arms (which can be controlled by fine-tuning the position of one of the coupling optics and can be used to scan the interferometer delay).

We now consider the beam splitter transformation

$$
\left(\begin{array}{l}
\hat{a}^{\dagger} \\
\hat{b}^{\dagger}
\end{array}\right) \rightarrow\left(\begin{array}{c}
\frac{1}{\sqrt{2}}\left(i \hat{a}^{\dagger}+\hat{b}^{\dagger}\right) \\
\frac{1}{\sqrt{2}}\left(\hat{a}^{\dagger}+i \hat{b}^{\dagger}\right)
\end{array}\right) .
$$

Using Eqs. (3) and (4), we obtain the two-photon state after the interaction with the beam splitter

$$
\begin{aligned}
\left|\chi_{\mathrm{bs}}\right\rangle= & \frac{1}{2} \int_{0}^{\infty} d \omega_{1} \int_{0}^{\infty} d \omega_{2} e^{-i\left\{\omega_{1}\left[t^{(+)}(f)+\delta t_{p}\right]+\omega_{2} t^{(-)}(f)\right\}} \\
& \times \psi\left(\omega_{1}, \omega_{2}\right)\left[i \hat{a}^{\dagger}\left(\omega_{1}\right)+\hat{b}^{\dagger}\left(\omega_{2}\right)\right] \\
& \times\left[\hat{a}^{\dagger}\left(\omega_{1}\right)+i \hat{b}^{\dagger}\left(\omega_{2}\right)\right]|0\rangle .
\end{aligned}
$$

We now consider the coincidence measurement

$\hat{\Pi}=\int_{0}^{\infty} d \omega_{3} \int_{0}^{\infty} d \omega_{4} \hat{a}^{\dagger}\left(\omega_{3}\right) \hat{b}^{\dagger}\left(\omega_{4}\right)|0\rangle\langle 0| \hat{a}\left(\omega_{3}\right) \hat{b}\left(\omega_{4}\right)$. 
Using Eqs. (5) and (6), it is then straightforward to obtain the probability $p\left(\delta t_{p}\right)=\left\langle\chi_{\mathrm{bs}}|\Pi| \chi_{\mathrm{bs}}\right\rangle$ of coincidence events. Specifically, we find

$$
\begin{aligned}
p\left(\delta t_{p}\right)= & \frac{1}{2}-\frac{1}{2} \int_{0}^{\infty} d \omega_{1} \int_{0}^{\infty} d \omega_{2} \psi\left(\omega_{2}, \omega_{1}\right)^{*} \psi\left(\omega_{1}, \omega_{2}\right) \\
& \times e^{i\left(\omega_{2}-\omega_{1}\right)\left[\Delta t(f)+\delta t_{p}\right]},
\end{aligned}
$$

where we have assumed $\int_{0}^{\infty} d \omega_{1} \int_{0}^{\infty} d \omega_{2}\left|\psi\left(\omega_{1}, \omega_{2}\right)\right|^{2}=1$, and $\Delta t=t^{(+)}-t^{(-)}$is the Sagnac delay given by Eq. (1). Note that Eq. (7) is the usual HOM coincidence probability but now with a dependence on rotational frequency $f$ in the time delay $\Delta t(f)$. For example, choosing $\psi\left(\omega_{1}, \omega_{2}\right)=$ $g\left(\omega_{1}\right) g\left(\omega_{2}\right)$, where $g(\omega) \approx\left(2 \pi \sigma^{2}\right)^{-1 / 4} e^{-(\omega-\mu)^{2} /\left(4 \sigma^{2}\right)}$ and $\mu \gg \sigma$, we find a simple expression

$$
p\left(\delta t_{p} ; f, A, \sigma\right)=\frac{1}{2}-\frac{1}{2} e^{-\sigma^{2}\left(\frac{8 \pi A f}{c^{2}}+\delta t_{p}\right)^{2}} .
$$

This result implies a linear relation between the measured delay (the position of the HOM dip in the coincidence count), the area $A$ of the interferometer, and the rotation frequency $f$. Moreover, the amplitude of the shift is exactly equal to that expected for a classical Sagnac interferometer.

Experimental results.-Experiments were performed by implementing the scheme shown in Fig. 1. To create a robust interferometer, rather than using free-space optics to form the clockwise and anticlockwise loops, we use a single mode polarization-maintaining fiber. The signal and idler beams are separated from each other using a knife-edge prism in the far field of the down-conversion crystal and both photons are subsequently coupled into the fiber, which forms the remainder of the optical system. A further advantage of a fiber-based approach is that a long length of fiber can be coiled to give many turns and hence increase the sensitivity of the shifts due to the rotation. This fiber-based approach could have been implemented using separate fibers for the clockwise and anticlockwise loops. However, separate fibers render the interferometer susceptible to drift due to unwanted temperature-based path-length variations, affecting each fiber differently. Here we insert additional beam splitters after the coupling optics to allow us to use the same fiber for both the clockwise and anticlockwise loops, albeit with a reduction in optical coupling efficiency.

Details of the actual experimental layouts are shown in Figs. 2(a) and 3(a): the fiber-based interferometer is composed of a $100 \mathrm{~m}$ long fiber wound $N=35$ times around a $0.908 \mathrm{~m}$ diameter loop. This provides an effective area of $A=N \pi r^{2}=22.7 \mathrm{~m}^{2}$. We first performed a classical experiment to calibrate the setup for the quantum measurements. We coupled a laser (642 nm wavelength) into the interferometer and measured the shifts in the spatial interference fringes on a CMOS camera at the output where the two counterpropagating signals overlap at a small angle.
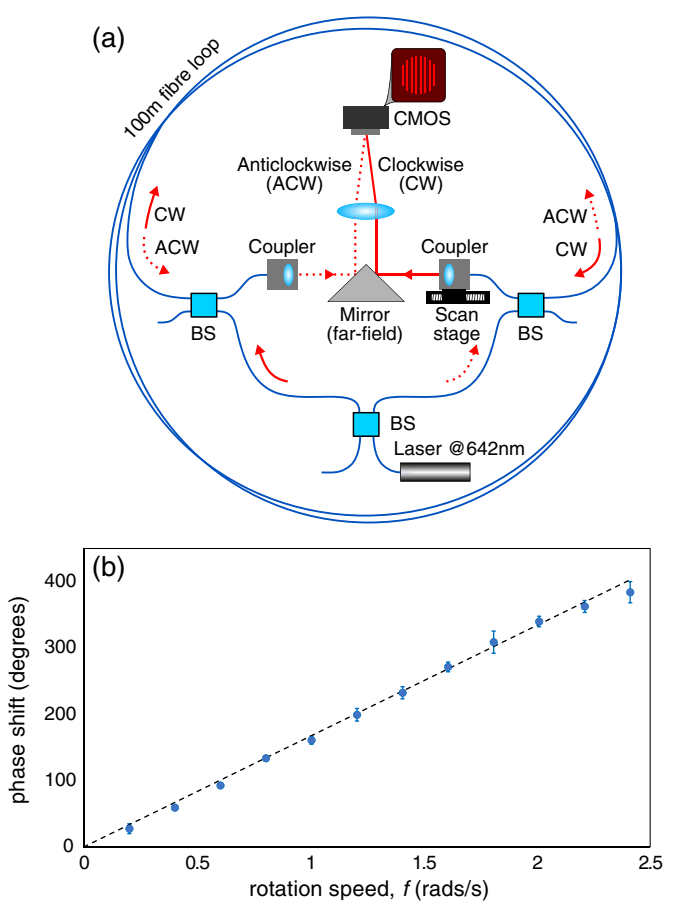

FIG. 2. Classical experiment. (a) Experimental layout. (b) Results showing measured Sagnac phase shifts (circles) and fitted line (dashed).

The results are shown in Fig. 2(b), where we plot the averaged measured phase shifts $\Delta \phi$ together with their standard deviation over 50 measurement runs. We also note that the phase shifts are obtained by performing the average (in absolute value) between a clockwise and an anticlockwise rotation measurement. This is in order to minimize the systematic deviations due to mechanical deformations caused by the presence of centrifugal acceleration $(\sim 0.3 g)$. The dashed line shows the best fit to the data with slope $\Delta \phi_{\text {fit }}=167 \pm 4 \mathrm{deg} \mathrm{Hz}^{-1}$. This is to be compared with the theoretical estimate based on the standard formula for the

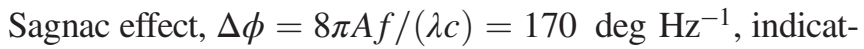
ing good agreement.

The HOM interference experiments were carried out with the same interferometer but with two modifications. We removed the laser and replaced it with two singlephoton avalanche detectors (SPADs), with which we then measured coincidence rates at the output of the HOM beam splitter. We also replaced the CMOS camera with a downconversion photon-pair source comprising a $355 \mathrm{~nm}$ pump laser and a barium borate, $\mathrm{BBO}$, crystal. The photon pairs are generated with an angular separation, both retracing the path of the classical setup, are separated by the prism, and interfere at the HOM beam splitter, as shown in Fig. 3(a). A delay stage on one of the fiber couplers provides a scan over the temporal delay: a typical result measured in the nonrotating case is shown in Fig. 3(b), with the typical "HOM dip" (a drop in coincidence rates) when the photons arrive simultaneously at the HOM beam splitter. 

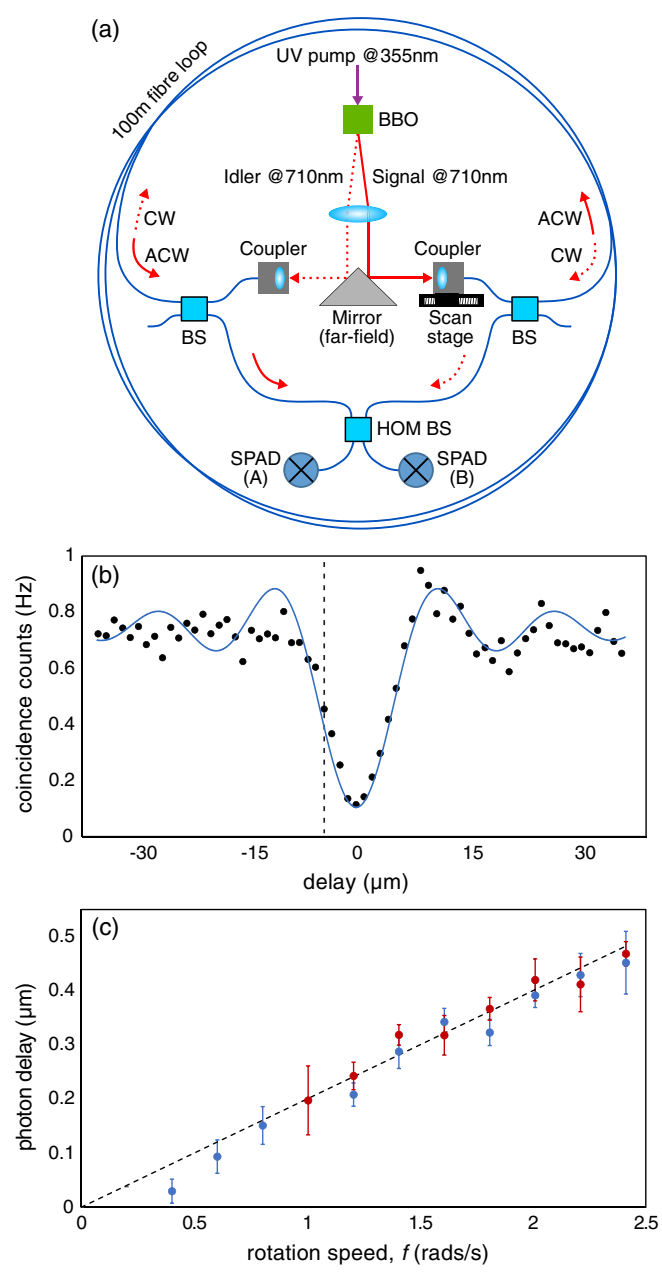

FIG. 3. Quantum experiment. (a) Experimental layout. (b) HOM interference dip measured by scanning the delay stage with no rotation of the experiment. The vertical dashed line marks the delay corresponding to the point of maximum steepness of the HOM dip. The shift can then be measured by fixing the stage at this delay and observing the changes in coincidence counts when the interferometer is in rotation. (c) Results showing measured HOM interference shift (circles) and fitted line (dashed).

A rotating HOM experiment involves comparing full scans of the HOM dip and analyzing the shift of its position as a function of rotation speed. This approach provides photon path delay measurements with a precision of the order of a micrometer. However, recent work [35] has shown that this precision can be greatly increased by fixing the interferometer delay close to the point of maximum steepness of the HOM interference dip, indicated by a vertical dashed line in Fig. 3(b). Shifts of the dip due to changes in photon path (in our case arising from rotation) are then measured by observing changes in the coincidence counts as the interferometer is rotated. Using the initially acquired HOM dip as a maximum-likelihood estimator, we can map the coincidence counts to photon path delay. This allows us to measure very small photon path delays down to $100 \mathrm{~nm}$ and smaller.

Figure 3(c) shows the mapped photon delays inferred from changes in coincidence counts, for increasing rotation rates, with data acquired over different days (blue/red circles). As for the classical experiment, we plot the average delays and standard deviations for 50 separate measurement runs and for the difference between clockwise and anticlockwise rotation. The dashed line shows the best fit to the data with slope $\Delta x_{\text {fit }}=200 \pm 12 \mathrm{~nm} \mathrm{~Hz}^{-1}$. These results clearly show that rotation can, for a fixed physical path length of the interferometer, modify the degree of distinguishability of two entangled photons and thus modify the quantum interference between the two photons. As such, this demonstrates a clear influence of noninertial motion on nonclassical photon states.

Discussion and conclusions.-We can compare the quantum and the classical results, for example, by taking the ratio of the slopes of the two fitted lines in Figs. 2(b) and 3(c): $\Delta x_{\text {fit }} / \Delta \phi_{\text {fit }}=1.2 \pm 0.07 \mathrm{~nm} \mathrm{deg}^{-1}$. Path and phase differences in an interferometer are related by $\Delta x / \Delta \phi=\lambda / 2 \pi$. If we use the vacuum wavelength for the classical laser $(642 \mathrm{~nm})$, we find that $\Delta x_{\text {fit }} / \Delta \phi_{\text {fit }}$ and $\Delta x / \Delta \phi$ differ by a multiplicative factor of $1.478 \pm 0.09$. This value is compatible with the refractive index $n \sim 1.45$ of the optical fiber, as may intuitively be expected based on the fact that the classical measurement is sensitive to phase (and does not depend on the fiber index $n$ [34]) whereas the quantum measurements rely on time delay (which does depend on $n$ ).

The experiments proposed here could be generalized to a scenario involving a satellite mission [36]. Specifically, instead of considering the Sagnac effect, we can consider its generalization to curved spacetime [37,38], i.e., the gravitomagnetic clock effect [31]. The rotating platform would be replaced by satellites orbiting around Earth: two photons would be transmitted in counterpropagating directions around the Earth, using three or more satellites, back to the satellite of origin, where they are interfered and detected. After a full revolution around the Earth, the difference in the arrival times of the two photons is given by the formula for the gravitomagnetic effect [39-41]: $\Delta t \sim G J /\left(R c^{4}\right)$, where $J$ is the angular momentum of the Earth, $R$ is the distance from its center, and $G$ is the gravitational constant. This relativistic effect would then modify the quantum interference for the two-photon state: the gravitationally induced photon delay would be of the order of $\Delta t \approx 10^{-16} \mathrm{~s}$. This delay is only 1 order of magnitude smaller than recent measurements based on HOM interferometry [35] and can be increased by increasing the number of revolutions around the Earth.

The measurement technique presented here builds upon recent developments in quantum sensing and constitutes a new approach to testing the interaction between quantum mechanics and special relativity, with routes toward the inclusion of nontrivial space and spacetime curvatures, for example, through nonuniform 
acceleration of the system or extension to gravitational fields with satellite-based experiments.

Open access data is available at Ref. [42].

We acknowledge discussions with Peter Horak and Giulio Gasbarri, and financial support from The Leverhulme Trust, the Foundational Questions Institute and EPSRC (UK Grants No. EP/P006078/2 and No. EP/R030081/1).

*These authors contributed equally to this work.

†daniele.faccio@glasgow.ac.uk

¥miles.padgett@glasgow.ac.uk

[1] R. Penrose, On gravity's role in quantum state reduction, Gen. Relativ. Gravit. 28, 581 (1996).

[2] R. Penrose, Experimental Search for Quantum Gravity, edited by S. Hossenfelder (Springer, New York, 2018).

[3] S. Hossenfelder, Approaches to Quantum Gravity: Toward a New Understanding of Space, Time and Matter, edited by D. Oriti (Cambridge University Press, Cambridge, United Kingdom, 2009).

[4] R. M. Wald, Quantum Field Theory in Curved Spacetime and Black Hole Thermodynamics (University of Chicago Press, Chicago, 1994).

[5] Advances in Algebraic Quantum Field Theory, edited by R. Brunetti, C. Dappiaggi, K. Fredenhagen, and J. Yngvason (Springer, New York, 2015).

[6] S. W. Hawking, Black hole explosions, Nature (London) 248, 30 (1974).

[7] S. W. Hawking, Black holes and thermodynamics, Phys. Rev. D 13, 191 (1976).

[8] S. Hossenfelder, Minimal length scale scenarios for quantum gravity, Living Rev. Relativity 16, 2 (2013).

[9] L. Diósi, Gravitation and quantum-mechanical localization of macro-objects, Phys. Lett. 105A, 199 (1984).

[10] R. Ruffini and S. Bonazzola, Systems of self-gravitating particles in general relativity and the concept of an equation of state, Phys. Rev. 187, 1767 (1969).

[11] D. Kafri, J. M. Taylor, and G. J. Milburn, A classical channel model for gravitational decoherence, New J. Phys. 16, 065020 (2014).

[12] L. Diósi, Models for universal reduction of macroscopic quantum fluctuations, Phys. Rev. A 40, 1165 (1989).

[13] A. Tilloy and L. Diósi, Sourcing semiclassical gravity from spontaneously localized quantum matter, Phys. Rev. D 93, 024026 (2016).

[14] G. Gasbarri, M. Toroš, S. Donadi, and A. Bassi, Gravity induced wave function collapse, Phys. Rev. D 96, 104013 (2017).

[15] R. Kaltenbaek et al., Macroscopic quantum resonators (maqro): 2015 update, EPJ Quantum Techno. 3, 5 (2016).

[16] R. Colella, A. W. Overhauser, and S. A. Werner, Observation of Gravitationally Induced Quantum Interference, Phys. Rev. Lett. 34, 1472 (1975).

[17] V. V. Nesvizhevsky et al., Quantum states of neutrons in the Earth's gravitational field, Nature (London) 415, 297 (2002).

[18] J. B. Fixler, G. T. Foster, J. M. McGuirk, and M. A. Kasevich, Atom interferometer measurement of the Newtonian constant of gravity, Science 315, 74 (2007).
[19] M. Fink, A. Rodriguez-Aramendia, J. Handsteiner, A. Ziarkash, F. Steinlechner, T. Scheidl, I. Fuentes, J. Pienaar, T. C. Ralph, and R. Ursin, Experimental test of photonic entanglement in accelerated reference frames, Nat. Commun. 8, 15304 (2017).

[20] D. N. Page and C. D. Geilker, Indirect Evidence for Quantum Gravity, Phys. Rev. Lett. 47, 979 (1981).

[21] C. Anastopoulos and B.-L. Hu, Probing a gravitational cat state, Classical Quantum Gravity 32, 165022 (2015).

[22] M. Derakhshani, C. Anastopoulos, and B.-L. Hu, Probing a gravitational cat state: Experimental possibilities, J. Phys. Conf. Ser. 701, 012015 (2016).

[23] T. Krisnanda, M. Zuppardo, M. Paternostro, and T. Paterek, Revealing Nonclassicality of Inaccessible Objects, Phys. Rev. Lett. 119, 120402 (2017).

[24] R. C. Ritter, C. E. Goldblum, W.-T. Ni, G. T. Gillies, and C. C. Speake, Experimental test of equivalence principle with polarized masses, Phys. Rev. D 42, 977 (1990).

[25] J. Schmöle, M. Dragosits, H. Hepach, and M. Aspelmeyer, A micromechanical proof-of-principle experiment for measuring the gravitational force of milligram masses, Classical Quantum Gravity 33, 125031 (2016).

[26] S. Bose, A. Mazumdar, G. W. Morley, H. Ulbricht, M. Toroš, M. Paternostro, A. A. Geraci, P. F. Barker, M. S. Kim, and G. Milburn, Spin Entanglement Witness for Quantum Gravity, Phys. Rev. Lett. 119, 240401 (2017).

[27] C. Marletto and V. Vedral, Gravitationally Induced Entanglement between Two Massive Particles is Sufficient Evidence of Quantum Effects in Gravity, Phys. Rev. Lett. 119, 240402 (2017).

[28] C.-K. Hong, Z.-Y. Ou, and L. Mandel, Measurement of Subpicosecond Time Intervals between Two Photons by Interference, Phys. Rev. Lett. 59, 2044 (1987).

[29] G. Sagnac, L'éther lumineux démontré par l'effet du vent relatif d'éther dans un interféromètre en rotation uniforme, Comptes Rendus Acad. Sci. 157, 708 (1913).

[30] G. Sagnac, Sur la preuve de la réalité de l'éther lumineux par l'expérience de l'interférographe tournant, Comptes Rendus Acad. Sci. 157, 1410 (1913).

[31] J. M. Cohen and B. Mashhoon, Standard clocks, interferometry, and gravitomagnetism, Phys. Lett. A 181, 353 (1993).

[32] Z.-Y. Ou and L. Mandel, Observation of Spatial Quantum Beating with Separated Photodetectors, Phys. Rev. Lett. 61, 54 (1988).

[33] É. Gourgoulhon, Special Relativity in General Frames: From Particles to Astrophysics (Springer, New York, 2016).

[34] E. J. Post, Sagnac effect, Rev. Mod. Phys. 39, 475 (1967).

[35] A. Lyons, G. C. Knee, E. Bolduc, T. Roger, J. Leach, E. M. Gauger, and D. Faccio, Attosecond-resolution Hong-Ou-Mandel interferometry, Sci. Adv. 4, eaap9416 (2018).

[36] F. Gronwald, E. Gruber, H. Lichtenegger, and R. A. Puntigam, Gravity Probe C(lock) — probing the gravitomagnetic field of the Earth by means of a clock experiment, arXiv:grqc/9712054. 
[37] M. L. Ruggiero and G. Rizzi, The relativistic Sagnac effect: Two derivations, in Relativity in Rotating Frames, edited by M. L. Ruggiero and G. Rizzi (Springer, Netherlands, 2003).

[38] M. L. Ruggiero, The Sagnac effect in curved space-times from an analogy with the Aharonov-Bohm effect, Gen. Relativ. Gravit. 37, 1845 (2005).

[39] A. Tartaglia, Detection of the gravitomagnetic clock effect, Classical Quantum Gravity 17, 783 (2000).
[40] I. Ciufolini and F. Ricci, Time delay due to spin inside a rotating shell, Classical Quantum Gravity 19, 3875 (2002).

[41] I. Ciufolini, S. Kopeikin, B. Mashhoon, and F. Ricci, On the gravitomagnetic time delay, Phys. Lett. A 308, 101 (2003).

[42] https://dx.doi.org/10.5525/gla.researchdata.852 (2019). 